\title{
Biomechanical Study of the Fixation Plates For Opening Wedge High Tibial Osteotomy
}

\author{
Kug Jin Kim, $\mathrm{MD}^{1}$, Eun Kyoo Song, $\mathrm{MD}^{2}$, Jong Keun Seon, $\mathrm{MD}^{2}$, and Jong Hwan Seol, $\mathrm{MD}^{2}$ \\ ${ }^{1}$ Department of Orthopedic Surgery, Wolchul Ki-Chan Hospital, Yeongam; ${ }^{2}$ Department of Orthopedic Surgery, Center for Joint Disease, Chonnam National University \\ Hwasun Hospital, Hwasun, Korea
}

Purpose: The purpose of this study was to compare the mechanical stability of three types of plate systems for opening wedge high tibial osteotomy. Materials and Methods: Forty-eight fresh frozen porcine tibia specimens were assigned to three different fixation device groups: Aescular group (16 specimens) was fixed with Aescular plates; Puddu group (16 specimens) with a Puddu plate, and TomoFix group (16 specimens) with a TomoFix plate. We compared axial displacements under compression loads from 200 to 2,000 N and maximal loads at failure among 8 specimens per group. We also compared displacements under cyclic load after 100 cycles at a compressive load of 2,000 $\mathrm{N}$ among 8 specimens per group.

Results: In all three groups, displacement under compression load increased with the increase in the axial compressive load; however, no significant intergroup differences were observed in the mean values under tested loading conditions. The mean maximal loads at failure were not significantly different $(6,055,6,798$, and 6,973 $\mathrm{N}$ in the Aescular, Puddu, and TomoFix groups, respectively; $\mathrm{p}=0.41)$. While the TomoFix group showed less extension and strain during the cyclic load test, the mean values showed no significant differences among groups.

Conclusions: All three plate systems were found to provide fixation stability suitable for bearing axial compression and cyclic loads while walking.

Keywords: Knee, High tibial osteotomy, Opening wedge, Fixation plate, Biomechanical study

\section{Introduction}

A number of surgical options are available for treating medial unicompartmental osteoarthritis (OA) of the knee, such as high tibial osteotomy (HTO), unicompartmental knee arthroplasty, and total knee arthroplasty. However, the activities of patients after arthroplasty may be limited and revision surgery is invariably required after 10 or 20 years. Accordingly, valgus HTO is becoming more popular for relatively active young patients with medial unicompartmental OA and varus deformity ${ }^{1-7)}$.

Opening wedge technique is becoming more popular because

Received December 10, 2014; Revised (1st) January 2, 2015;

Accepted January 29, 2015

Correspondence to: Jong Hwan Seol, MD

Department of Orthopedic Surgery, Chonnam National University

Hwasun Hospital, 322 Seoyang-ro, Hwasun 58128, Korea

Tel: +82-61-379-7676, Fax: +82-61-379-7681

E-mail:kssul5@naver.com

This is an Open Access article distributed under the terms of the Creative Commons Attribution Non-Commercial License (http://creativecommons.org/licenses/by-nc/4.0/) which permits unrestricted non-commercial use, distribution, and reproduction in any medium, provided the original work is properly cited. it has some advantages over closing wedge osteotomy. It has been shown that adequate stable fixation is mandatory for safe healing of the osteotomy to minimize the risk of nonunion and loss of correction after medial opening wedge $\mathrm{HTO}^{8}$. Some special implants have been developed in an attempt to make medial opening wedge HTO procedure stable.

The Puddu plate (Arthrex Inc., Naples, FL, USA) is the most widely used fixation device for the medial opening HTO. However, several studies have reported higher complication rates of nonunion, fixation failure, and correction loss, due to unstable fixation in medial opening HTO using the Puddu plate ${ }^{9,10)}$. Hence, internal fixators such as TomoFix plate (Synthes, Oberdorf, Switzerland) and Aescular plate (B. Braun Korea, Seoul, Korea) were developed to achieve optimal stability and maintain the attained correction.

There are several comparative studies regarding the comparative fixation strength of theses implants. Stoffel et al. ${ }^{11)}$ suggested that the TomoFix plate would provide superior stability under both compression and torsion compared to the Puddu plate in a situation where the lateral cortex is damaged. Jung et al. $^{8)}$ insisted that loss of correction after opening-wedge HTO using the 


\section{Kim et al. Open Wedge HTO: Comparative Mechanical Stability}

Aescular plate was significant compared with the TomoFix plate. However, there is no biomechanical study comparing all three implants.

This study was undertaken to compare the mechanical stability of the three fixation plate systems for opening wedge HTO based on the mechanical test results obtained using a porcine tibia model. Our hypothesis was that the Aescular plate would show no difference compared to other fixation plate systems with respect to mechanical stability.

\section{Materials and Methods}

\section{Specimen Preparation}

Fifty-four tibias from 1-year-old domestic pigs weighting 90$100 \mathrm{~kg}$ were used. Specimens were stored at $-70^{\circ} \mathrm{C}$ until required and thawed at room temperature for $24 \mathrm{hrs}$ before experiments. The soft tissues were completely removed, but fibular and tibiofibular joints (anterior, posterior tibiofibular ligament and interosseous membrane) were spared. A medial opening wedge osteotomy was performed at $20 \mathrm{~mm}$ below the tibial plateau targeting the tibiofibular joint using an oscillating saw. The osteotomy was opened carefully using a small osteotome and an osteotomy wedge was formed gradually so as not to break the lateral cortex of the tibia. All osteotomies were performed by one surgeon using standard techniques. After $10 \mathrm{~mm}$ opening of the osteotomy site, fixation was carried out on the osteotomy site using either Aescular plate (B. Braun Korea), a Puddu plate (Arthrex Inc.), or a TomoFix plate (Synthes) without a bone graft.

In the Aescular group, two plate sizes were used, that is, the anterior plate was smaller than the posterior plate to prevent subsequent increases in posterior slope. Two $6.5 \mathrm{~mm}$ cancellous screws were used for each plate fixation. In the Puddu group, two 6.5 $\mathrm{mm}$ cannulated screws were used for proximal fixation and two $4.5 \mathrm{~mm}$ cortical screws for distal fixation. In the TomoFix group, osteotomy was fixed with plates and $4.5 \mathrm{~mm}$ locking screws. Three $4.5 \mathrm{~mm}$ locking screws were used for proximal fixation and four $4.5 \mathrm{~mm}$ locking screws for distal fixation. The ends of both plates were then molded with bone cement for mechanical testing (Fig. 1). This process was performed using a jig that aligned the long axis of the tibia with the axis of the Instron 5569 series unit (Instron, Norwood, MA, USA) (Fig. 2).

Six of the original 54 specimens were excluded due to lateral cortical breakage during osteotomy or the opening procedure. Thus, 48 fresh-frozen specimens were assigned to the three study

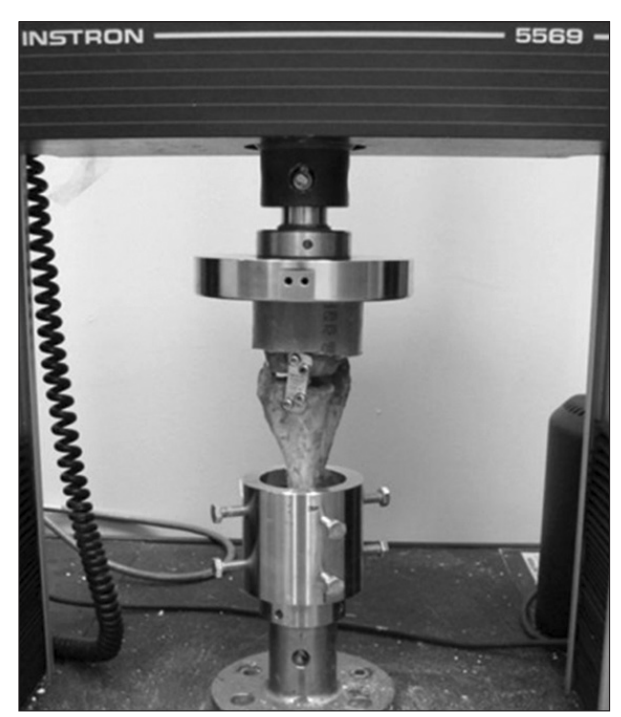

Fig. 2. Testing arrangement with axial loading of opening wedge high tibial osteotomy using mechanical testing system (Instron).
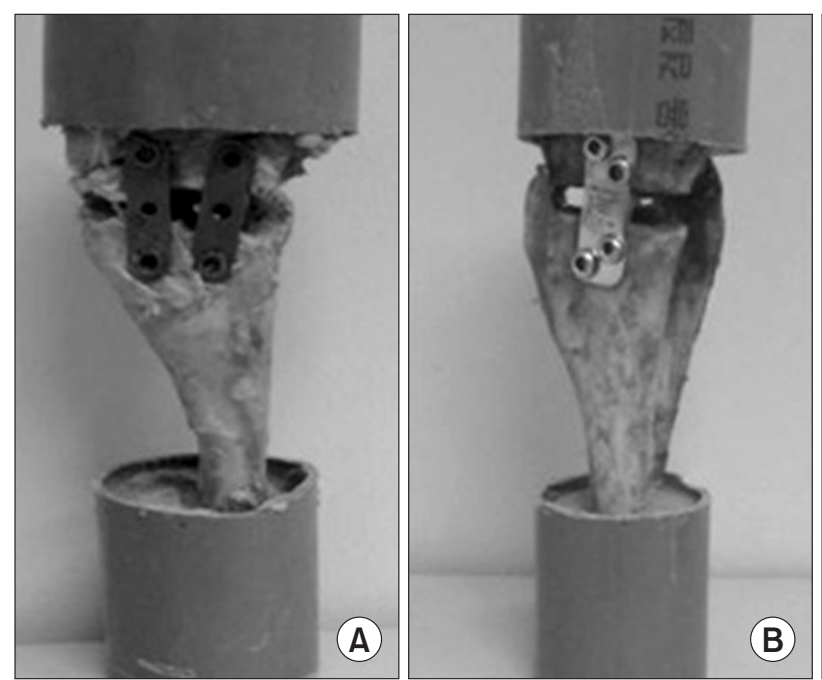

Fig. 1. Three plates for fixation of open wedge high tibial osteotomy: (A) Aescular plate, (B) Puddu plate, and (C) TomoFix plate. 
groups, that is, Aescualr group (16 specimens) fixed with Aescular plates; Puddu group (16 specimens) with a Puddu plate, and TomoFix group (16 specimens) with a TomoFix plate.

\section{Mechanical Testing}

Axial compression load testing and compression-extension cyclic load testing were both conducted on 8 tibias per group. Initially, a preload of $50 \mathrm{~N}$ was applied for 3 minutes before axial compression. The width of the ventral and dorsal parts of the osteotomy space was measured by micrometer (tolerance $0.1 \mathrm{~mm}$ ) during this time interval. The difference between the averages of the two values was defined as displacement. The point for measurement was marked with a water proof fine liner on the ventral and dorsal tibial edges. Axial compression load testing involved a gradual axial compression load test and a maximal compression axial load test. Axial displacement was measured by increasing the axial compression force in steps from 200 to 2,000 N (200, $500,1,000$, and $2,000 \mathrm{~N})^{12}$. Specimens were then loaded to failure to determine maximal failure loads; testing was stopped when loads fell by $30 \%$ of maximum loads (Fig. 3). Maximal load and displacement were measured at the time of failure. The testing machine was operated at a constant speed of $20 \mathrm{~mm} / \mathrm{min}$ for all tests.

Compression-extension cyclic load testing involved the cyclic application of compressive force and an extension force from 0 to 2,000 N. Extension means distraction of proximal tibia. Loading was applied to the tibial shaft for 100 cycles at $1 \mathrm{~Hz}$, and the loading condition was from 0 to 2,000 $\mathrm{N}^{13}$. Extension length $(\mathrm{mm})$ and strain (\%) were recorded during the last complete test cycle. Extension length was recorded as the difference of length between the initial cycle and the last cycle. Strain was defined as subjective amount of displacement. So, strain was calculated as the extension length divided by the initial length. A compressive load of 2,000 N corresponds to the load exerted on the knee by a person weighing $70 \mathrm{~kg}$ during normal walking. Test control and data collection were performed using MAX software (Instron).

\section{Statistical Analysis}

Analysis of variance was used to compare the three groups with respect to displacement and loads under compression and cyclic loads. The parameters are given as the mean \pm standard deviation. SPSS ver. 17.0 (SPSS Inc., Chicago, IL, USA) was used to compare results, and statistical significance was accepted for p-values of $<0.05$.

\section{Results}

\section{Displacement in Axial Compression Load Test}

As axial compressive load was gradually applied, plate displacements increased in all three groups. In the Aescular group, the osteotomy model showed a mean displacement of $0.27 \pm 0.16 \mathrm{~mm}$ at $200 \mathrm{~N}$, but this increased to $2.70 \pm 1.38 \mathrm{~mm}$ at $2,000 \mathrm{~N}$. In the Puddu group, the osteotomy model showed a mean displacement of $0.17 \pm 0.14 \mathrm{~mm}$ at $200 \mathrm{~N}$, which increased to $2.08 \pm 1.16 \mathrm{~mm}$ at $2,000 \mathrm{~N}$. The TomoFix group showed a displacement of $0.15 \pm 0.09$ $\mathrm{mm}$ at $200 \mathrm{~N}$ and $1.81 \pm 1.06 \mathrm{~mm}$ at $2,000 \mathrm{~N}$. All three groups showed similar increases in displacement with the increase of axial compressive loads (Table 1), but no significant intergroup difference in mean values was observed under different amount of loading conditions $(\mathrm{p}>0.05)$.
A

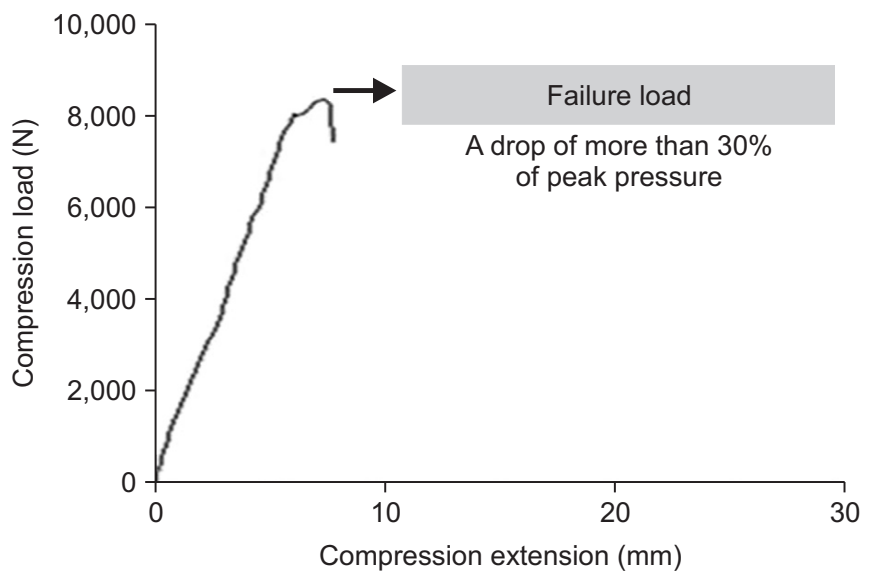

B

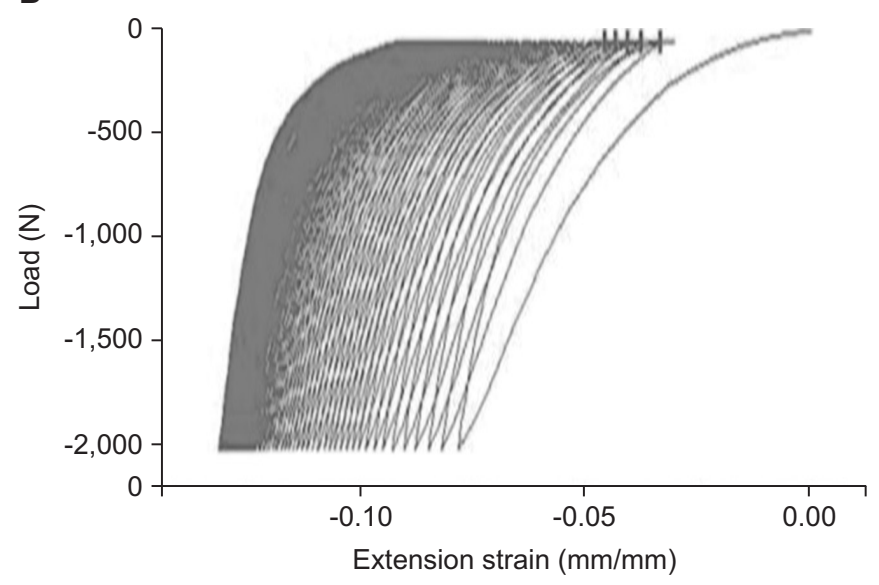

Fig. 3. (A) Graph of axial compression load test. Failure load was defined as a drop of more than $30 \%$ of peak pressure. (B) Graph of compression/ extension cyclic load test (2,000 $\mathrm{N}$ compression load). 


\section{Kim et al. Open Wedge HTO: Comparative Mechanical Stability}

The maximum load at the time of failure was $6,055.1 \pm 1,184.7 \mathrm{~N}$ in the Aescular group, 6,798.2 $\pm 998.7 \mathrm{~N}$ in the Puddu group, and $6,973.8 \pm 499.7 \mathrm{~N}$ in the TomoFix group. At the time of failure, the Aescular, Pudda, and Tomofix groups showed a displacement of $7.54 \pm 2.32 \mathrm{~mm}, 7.31 \pm 2.05 \mathrm{~mm}$, and $6.41 \pm 3.31 \mathrm{~mm}$, respectively (Fig. 4). However, no significant difference was found in the mean maximal loads or displacements at the time of failure among three groups $(\mathrm{p}=0.41, \mathrm{p}=0.41)$.

\section{Compression-Extension Cyclic Load Test}

In the Aescular, Puddu, and TomoFix groups, the mean extension under cyclic loading test was $2.91 \pm 0.39 \mathrm{~mm}, 2.56 \pm 0.38 \mathrm{~mm}$, and $2.02 \pm 0.27 \mathrm{~mm}$, respectively, and corresponding mean strain (\%) was $9.20 \pm 1.23,8.08 \pm 1.20$, and $6.38 \pm 0.86$, respectively (Table 2). The mean extension and strain were slightly smaller in the TomoFix group than the other two groups. However, no significant differences were observed among groups in terms of extension $(\mathrm{p}=0.21)$ and strain $(\mathrm{p}=0.43)$

Table 1. Displacement in Axial Compression Load Test according to the Type of Plate

\begin{tabular}{lcccc}
\hline \multirow{2}{*}{ Loading $(\mathrm{N})$} & \multicolumn{3}{c}{ Type of plate $(\mathrm{mm})$} & \multirow{2}{*}{ p-value } \\
\cline { 2 - 4 } & Aescular & Puddu & TomoFix & \\
\hline 200 & $0.27 \pm 0.16$ & $0.17 \pm 0.15$ & $0.15 \pm 0.09$ & 0.534 \\
500 & $0.62 \pm 0.40$ & $0.49 \pm 0.41$ & $0.52 \pm 0.43$ & 0.458 \\
1,000 & $1.26 \pm 0.77$ & $0.95 \pm 0.67$ & $0.91 \pm 0.61$ & 0.433 \\
2,000 & $2.70 \pm 1.38$ & $2.08 \pm 1.16$ & $1.81 \pm 1.06$ & 0.471 \\
\hline
\end{tabular}

Values are presented as mean \pm standard deviation.

A

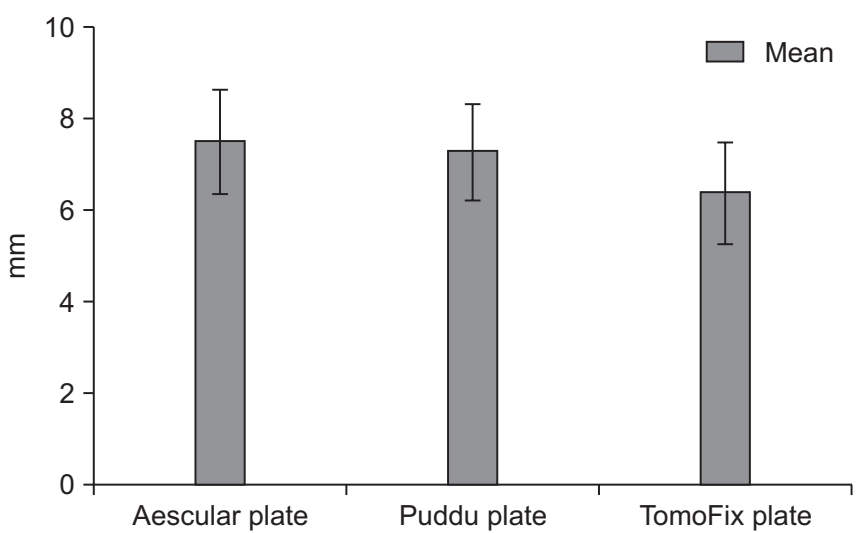

\section{Discussion}

Opening wedge osteotomy has higher complication rates than closing wedge osteotomy with respect to fixation failure, loss of correction, nonunion, and delayed union ${ }^{10,14)}$. In particular, the rate of nonunion has been reported to be higher for opening wedge HTO than closing wedge HTO, ranging between $10 \%$ and $35 \%$. Furthermore, after medial opening wedge osteotomy, the gap created in the proximal tibia facilitates instability and loss of correction, the latter of which is mainly caused by a lack of primary stability ${ }^{15)}$. Meidinger et al. ${ }^{16)}$ reported a $5.4 \%$ nonunion rate (10 of 182 patients) for medial opening wedge HTO, and concluded that lateral cortex preservation and secure fixation were most important in terms of preventing complications after opening wedge HTO. In another study, inadequate fixation stability of opening wedge plates was found to be a reason for opening wedge HTO failure ${ }^{17)}$.

Of the implants specifically designed for medial opening wedge HTO, Puddu plates are most commonly used. This plate is fixed to the medial side of the tibial plateau, and a spacer is inserted into the osteotomy gap, thus preventing gap closure. However, some authors have reported high failure rates of this implant and technique in HTO due to unstable fixation ${ }^{11,18)}$. For these reasons,

Table 2. The Extension Values and Strain Degrees according the Type of Plate in Compressive/Extension Cyclic Load Test

\begin{tabular}{lccc}
\hline \multicolumn{1}{c}{ Variable } & Aescular plate & Puddu plate & TomoFix plate \\
\hline Extension $(\mathrm{mm})$ & $2.91 \pm 0.39$ & $2.56 \pm 0.38$ & $2.02 \pm 0.27$ \\
Strain $(\%)$ & $9.20 \pm 1.23$ & $8.08 \pm 1.20$ & $6.38 \pm 0.86$ \\
\hline
\end{tabular}

Values are presented as mean \pm standard deviation.

Under 2,000 N, 100 cycles of compressive/extension cyclic load.

B

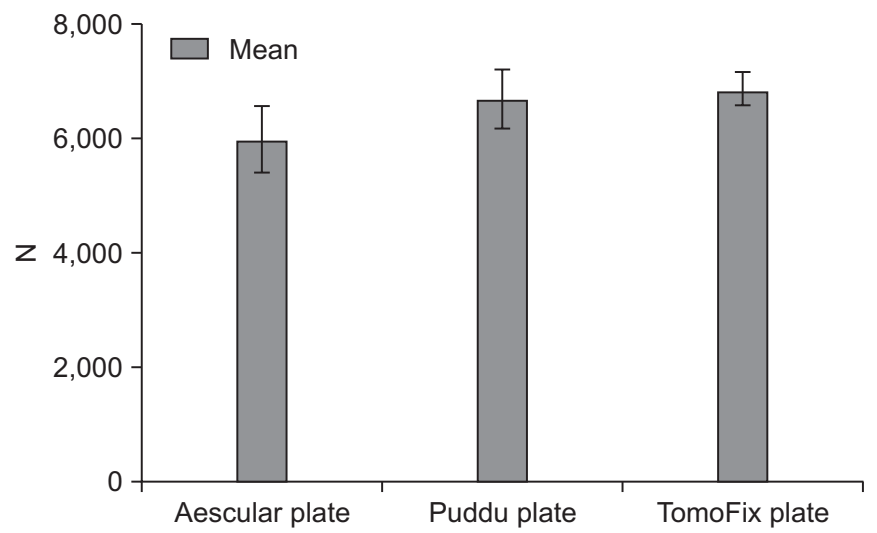

Fig. 4. (A) Degree of displacement at the time of failure. (B) Maximum load at the time of failure. 
a new implant with a long, rigid titanium plate (TomoFix) with the curvature of the medial tibial metaphysis was designed for opening wedge HTO.

Stoffel et al. ${ }^{11)}$ examined the construct stiffness and fixation strengths of the Puddu and TomoFix plates, and found that both were able to maintain correction at a load of more than $2,500 \mathrm{~N}$, which concurs with our results. This finding suggests that both plates can provide sufficient stability to maintain an axial compressive load in the knee of an adult in a single limb stance or during walking ${ }^{19)}$. However, TomoFix plates were found to provide better stability in both compression and torsion than Puddu plates when the lateral cortex was fractured. In the present study, mean displacements of 2.08 and $2.13 \mathrm{~mm}$ were observed for Puddu and TomoFix plates, respectively, at a compressive load of $2,000 \mathrm{~N}$, indicating no significant difference between the plates. However, TomoFix plates showed better stability during cyclic load testing ( $2.5 \mathrm{~mm}$ vs. $2.0 \mathrm{~mm}$ displacement for Puddu and TomoFix plates, respectively).

Although TomoFix plates provide sufficient initial stability after opening wedge osteotomy, they are too large if a small incision is required and the posterior tibial slope is not easily controlled. To resolve these problems, an opening wedge plate system consisting of two plates (Aescular plate; B. Braun Korea) was developed. This system consists of anterior and posterior plates; the posterior plate is placed as posteriorly as possible at the corner of the proximal tibia, and the anterior plate is placed behind the tibial tuberosity. Thus, surgeons can control the tibial slope and prevent an unintended increase in posterior tibial slope. However, the fixation stability of this fixation device has not been fully established. In this study, primary fixation stability of this plate was compared with those of the other two plates. The amount of displacement of the Aescular plate during axial compression load testing was not significantly different from the other two plates. Maximal loads at failure were 6,055.1 $\pm 1,184.7 \mathrm{~N}, 6,798.2 \pm 988.7$ $\mathrm{N}$, and $6,973.8 \pm 499.8 \mathrm{~N}$ for the Aescular, Puddu, and TomoFix plates, respectively, which were also not significantly different. All three plates were able to maintain correction at loads of greater than 2,500 N, which approximates to the axial compression load in the knee of an adult during walking ${ }^{19)}$.

During cyclic load testing using 100 cycles of a 2,000 N compressive force, the mean displacement of the Aescular system was comparable to that of the Puddu system (2.91 mm vs. 2.56 $\mathrm{mm})$. However, the TomoFix system exhibited greater stability $(2.02 \mathrm{~mm})$ than the Aescular and Puddu systems, although the intergroup differences in the mean displacement were not significant and less than $1 \mathrm{~mm}$ during the cyclic load test. These results showed that the primary stability of three systems were similar each other under cyclic loading.

There are some limitations of this study. First of all, we fully acknowledge that the porcine bone could have different mechanical properties to a human bone even though we performed a bone quality test using quantitative computed tomography. Second, the osteotomy site of HTO is broad; therefore, there can be a little difference of displacement in the medial and lateral osteotomy sites. We are aware that we did not consider the difference of displacement in the medial and lateral osteotomy sites so that the result of difference in medial and lateral side displacement was not taken into consideration. In addition, we evaluated only displacement of the osteotomy site. Torsion is one of major factors for biomechanical studies on plates. But we did not address the results of torsion in this study. Third, the study was aimed at evaluating the stability of the plates during walking; however, we used Instron instead of the femur for the upper loading, and were unable to accurately simulate the walking condition. Forth, this study only dealt with an axial load without any other load components.

\section{Conclusions}

Based on the comparison of stability of three types of fixation plate systems, the Aescular, Puddu, and TomoFix plate systems were found to provide fixation sufficient stability to bear axial compression and cyclic loads during walking.

\section{Conflict of Interest}

No potential conflict of interest relevant to this article was reported.

\section{References}

1. Brinkman JM, Lobenhoffer P, Agneskirchner JD, Staubli AE, Wymenga $A B$, van Heerwaarden RJ. Osteotomies around the knee: patient selection, stability of fixation and bone healing in high tibial osteotomies. J Bone Joint Surg Br. 2008; 90:1548-57.

2. Backstein D, Meisami B, Gross AE. Patella baja after the modified Coventry-Maquet high tibial osteotomy. J Knee Surg. 2003;16:203-8.

3. Flecher X, Parratte S, Aubaniac JM, Argenson JN. A 12-28year followup study of closing wedge high tibial osteotomy. Clin Orthop Relat Res. 2006;452:91-6.

4. Majima T, Yasuda K, Katsuragi R, Kaneda K. Progression of 
joint arthrosis 10 to 15 years after high tibial osteotomy. Clin Orthop Relat Res. 2000;(381):177-84.

5. Odenbring S, Tjornstrand B, Egund N, Hagstedt B, Hovelius L, Lindstrand A, Luxhoj T, Svanstrom A. Function after tibial osteotomy for medial gonarthrosis below aged 50 years. Acta Orthop Scand. 1989;60:527-31.

6. Salzmann GM, Ahrens P, Naal FD, El-Azab H, Spang JT, Imhoff $\mathrm{AB}$, Lorenz S. Sporting activity after high tibial osteotomy for the treatment of medial compartment knee osteoarthritis. Am J Sports Med. 2009;37:312-8.

7. van Raaij T, Reijman M, Brouwer RW, Jakma TS, Verhaar JN. Survival of closing-wedge high tibial osteotomy: good outcome in men with low-grade osteoarthritis after 10-16 years. Acta Orthop. 2008;79:230-4.

8. Jung WH, Chun CW, Lee JH, Ha JH, Kim JH, Jeong JH. Comparative study of medial opening-wedge high tibial osteotomy using 2 different implants. Arthroscopy. 2013;29:1063-71.

9. Amendola A. Unicompartmental osteoarthritis in the active patient: the role of high tibial osteotomy. Arthroscopy. 2003; 19 Suppl 1:109-16.

10. Gaasbeek RD, Welsing RT, Verdonschot N, Rijnberg WJ, van Loon CJ, van Kampen A. Accuracy and initial stability of open- and closed-wedge high tibial osteotomy: a cadaveric RSA study. Knee Surg Sports Traumatol Arthrosc. 2005; 13:689-94.

11. Stoffel K, Stachowiak G, Kuster M. Open wedge high tibial osteotomy: biomechanical investigation of the modified Arthrex Osteotomy Plate (Puddu Plate) and the TomoFix Plate. Clin Biomech (Bristol, Avon). 2004;19:944-50.
12. Spahn G, Muckley T, Kahl E, Hofmann GO. Biomechanical investigation of different internal fixations in medial opening-wedge high tibial osteotomy. Clin Biomech (Bristol, Avon). 2006;21:272-8.

13. Takeuchi R, Bito H, Akamatsu Y, Shiraishi T, Morishita S, Koshino T, Saito T. In vitro stability of open wedge high tibial osteotomy with synthetic bone graft. Knee. 2010;17:217-20.

14. Miller BS, Downie B, McDonough EB, Wojtys EM. Complications after medial opening wedge high tibial osteotomy. Arthroscopy. 2009;25:639-46.

15. Jackson JP, Waugh W. Tibial osteotomy for osteoarthritis of the knee. J Bone Joint Surg Br. 1961;43:746-51.

16. Meidinger G, Imhoff AB, Paul J, Kirchhoff C, Sauerschnig M, Hinterwimmer S. May smokers and overweight patients be treated with a medial open-wedge HTO? Risk factors for non-union. Knee Surg Sports Traumatol Arthrosc. 2011; 19:333-9.

17. Brouwer RW, Bierma-Zeinstra SM, van Raaij TM, Verhaar JA. Osteotomy for medial compartment arthritis of the knee using a closing wedge or an opening wedge controlled by a Puddu plate. A one-year randomised, controlled study. J Bone Joint Surg Br. 2006;88:1454-9.

18. Flamme CH, Kohn D, Kirsch L. High tibial osteotomy: primary stability of several implants. Z Orthop Ihre Grenzgeb. 1999;137:48-53.

19. Matthews LS, Goldstein SA, Malvitz TA, Katz BP, Kaufer H. Proximal tibial osteotomy. Factors that influence the duration of satisfactory function. Clin Orthop Relat Res. 1988; (229):193-200. 